Click

Here

Full

Article

\title{
Improved estimates of percolation and anisotropic permeability from 3-D X-ray microtomography using stochastic analyses and visualization
}

\section{Jie Liu}

Computational Geoscience, CSIRO Exploration and Mining, P.O. Box 1130, Bentley, Western Australia 6102, Australia (jie.liu@csiro.au)

\section{Klaus Regenauer-Lieb}

Computational Geoscience, CSIRO Exploration and Mining, P.O. Box 1130, Bentley, Western Australia 6102, Australia

Also at School of Earth and Geographical Sciences, University of Western Australia, 35 Stirling Highway, Perth, Western Australia 6009, Australia

\section{Chris Hines}

iVEC, P.O. Box 1130, Bentley, Western Australia 6102, Australia

\section{Keyu Liu}

CSIRO Petroleum, P.O. Box 1130, Bentley, Western Australia 6102, Australia

\section{Oliver Gaede}

School of Earth and Geographical Sciences, University of Western Australia, 35 Stirling Highway, Perth, Western Australia 6009, Australia

\section{Andrew Squelch \\ iVEC, P.O. Box 1130, Bentley, Western Australia 6102, Australia \\ Also at Department of Exploration Geophysics, Curtin University of Technology, GPO Box U1987, Perth, Western Australia 6845, Australia}

[1] X-ray microtomography (micro-CT) with micron resolution enables new ways of characterizing microstructures and opens pathways for forward calculations of multiscale rock properties. A quantitative characterization of the microstructure is the first step in this challenge. We developed a new approach to extract scale-dependent characteristics of porosity, percolation, and anisotropic permeability from 3-D microstructural models of rocks. The Hoshen-Kopelman algorithm of percolation theory is employed for a standard percolation analysis. The anisotropy of permeability is calculated by means of the star volume distribution approach. The local porosity distribution and local percolation probability are obtained by using the local porosity theory. Additionally, the local anisotropy distribution is defined and analyzed through two empirical probability density functions, the isotropy index and the elongation index. For such a high-resolution data set, the typical data sizes of the CT images are on the order of gigabytes to tens of gigabytes; thus an extremely large number of calculations are required. To resolve this large memory problem parallelization in OpenMP was used to optimally harness the shared memory infrastructure on 
cache coherent Non-Uniform Memory Access architecture machines such as the iVEC SGI Altix 3700Bx2 Supercomputer. We see adequate visualization of the results as an important element in this first pioneering study.

Components: 5674 words, 11 figures, 5 tables.

Keywords: X-ray microtomography; percolation; anisotropic permeability; stochastic analysis; visualization; parallelization.

Index Terms: 5112 Physical Properties of Rocks: Microstructure; 5114 Physical Properties of Rocks: Permeability and porosity; 3265 Mathematical Geophysics: Stochastic processes $(3235,4468,4475,7857)$.

Received 11 December 2008; Revised 2 April 2009; Accepted 13 April 2009; Published 29 May 2009.

Liu, J., K. Regenauer-Lieb, C. Hines, K. Liu, O. Gaede, and A. Squelch (2009), Improved estimates of percolation and anisotropic permeability from 3-D X-ray microtomography using stochastic analyses and visualization, Geochem. Geophys. Geosyst., 10, Q05010, doi:10.1029/2008GC002358.

\section{Introduction}

[2] In this contribution we make use of the increasing power of X-ray micro-CT which is now available at submicron resolution for some desktop devices and X-ray synchrotron techniques. The technology has potential to revolutionize the analysis of rock textures, an excellent review on the state of the art is given by Jerram and Higgins [2007]. We process the 3-D images with the improved computer processing capability of a modern parallel computer system. Our goal is to extract a statistical scale-dependent quantification of microstructures to be used for further numerical modeling for larger-scale processes such as fluid flow through fractured porous rocks. We propose to combine our stochastic description with other techniques in the future to extract transport and other physical properties from microtomographic images by forward numerical simulation [Arns et al., 2001; Knackstedt et al., 2006]. In order to achieve this goal we extend the original formulation of the local porosity theory [Hilfer, 1992, 2002] to include the quantification of anisotropic permeability microstructure. The resulting algorithm is a powerful method that is not restricted to stochastic analysis of permeability but can be used to derive inputs for a stochastic geometric model of any anisotropic microstructures.

[3] The local porosity theory [Hilfer, 1992, 2002] in its basic application extracts just two geometric characteristics, the local porosity distribution and the local percolation probability. It provides a scale-dependent characterization of the microstructure of porous media that can be used as an effective medium approach to predict fluid flow transport [Biswal et al., 1998; Widjajakusuma et al., 1999; Biswal and Hilfer, 1999; Manwart et al., 2002]. Anisotropy of permeability is one of the most important transport properties influencing fluid flow in 3-D space, but it is not included in the original local porosity theory. Ketcham and Iturrino [2005] analyzed and visualized the anisotropy of permeability using a high-resolution X-ray CT data set, in order to derive the distribution of effective porosity for permeability analysis. They used an approach where the sample were imaged twice; once with its pore space empty (in dry sample) and second with the pore saturated with water (in wet sample). By subtracting the raw CT images of the saturated and dry samples from each other the pore space was identified. This experimental approach, however, was expensive and inconvenient. In addition, the results are subject to subtle changes in environmental conditions and operational processes.

[4] Parallel computing of percolation is the field of computer sciences, physics and mathematics (D. W. Blair and J. Machta, The parallel computational complexity of the percolation model, paper presented at APS March Meeting, American Physical Society, Denver, Colorado, 2007, available at http://meetings.aps.org/link/BAPS.2007.MAR. C1.90). To the best of our knowledge no parallel computer algorithms for local porosity theory have been published. Therefore, analyses are currently restricted by the memory of the computer. Considering the typical file size of several tens of gigabytes this analysis cannot be performed on standard computer architecture. We present a technique that overcomes this limitation. Our codes are parallelized in OpenMP and the speed-up is tested on the iVEC SGI Altix 3700Bx2. We also visualize the 3-D reconstruction by using AMIRA $^{\mathbb{R}}$ for 
microstructures, GID ${ }^{\circledR}$ for cluster visualization, and the open software Mayavi for tensor visualization.

[5] This method is our first fundamental step for understanding the upscaling from microscale to macroscale fluid flow. For homogeneous media, an essential representative element volume can be extracted on the basis of the scale-dependent properties of fluid transport for upscaling. Our ultimate purpose is to derive the relation between fluid flow and deformation at large crustal scale in a multiscale earth systems approach [Regenauer-Lieb et al., 2008]. For general natural heterogeneous models, this method could be used as a building block for a heterogeneous multiscale method $[E$ and Engquist, 2003] by feeding microscale observation data to the microscale model.

\section{Methodology}

\subsection{Data Preparation: From CT Images to 3-D Label Data}

[6] Our working data is a stack of gray scale images which is obtained from the reconstructed data of CT scanning. We use the Amira ${ }^{\mathbb{R}}$ software package to process the images. The software package enables us to (1) build up 3-D microstructure which is a procedure from pixels in image slices to voxels in a volume; (2) crop any parallelepiped from the 3-D volume for our interest or as a representative volume; (3) resample the data to get coarser resolution for reducing computation time and improving rendering performances, as an option; (4) implement image segmentation which separates our target fabric or structure from the matrix, e.g., separates pores from solids in porous media; and (5) visualize the microstructure in different styles.

[7] A crucial procedure for our next analysis is to export a label data based on the above processes. The label data is a file that stores the data of the cropped 3-D parallelepiped after segmentation, in which voxels that have a value between the specific threshold values are labeled as 0 and others are labeled as 1 . For example, pores are labeled as 0 and solids are 1 in porous media. All our analyses in the following use only the label data.

\subsection{Extraction of Parameters From a 3-D Model}

[8] Three parameters are extracted from the microstructure model, including porosity, percolation and anisotropy of permeability. Porosity $\varphi$ is simply defined as the ratio of the volume of pore space to the total (or bulk) volume. Both volumes can be calculated from the label data of the 3-D model.

[9] In mathematics and physics, percolation generally refers to the nature of the connectivity in lattice models of random systems. In geological terms, percolation is defined as the slow movement of fluids through a porous material. Here we use the mathematical definition of percolation. To derive the percolation analysis of a model, two terms are defined: (1) neighbors are voxels with one common plane in the 3-D microstructural model of the cubic lattice and (2) a cluster is a group of neighbors which are connected to each other with the same material label. Then two steps, labeling clusters and evaluating percolation, are implemented. Labeling clusters is a process of giving all voxels within the same cluster the same label. This is a time consuming step for a large data set, thus the Hoshen-Kopelman algorithm [Hoshen and Kopelman, 1976] is used to reduce the computing time. We define a given direction is percolating when at least one voxel on one side of a sample volume has the same label as at least one voxel on the opposite side [Stauffer and Aharony, 1994].

[10] The anisotropy of permeability is calculated from the star volume distribution method following Ketcham [2005], on the basis of the assumption that permeability anisotropy is in part a function of directional variations in the character of the pore structure. The pore structure is represented by the structure of the percolating cluster. For the cluster which consists of a set of $n$ sites (voxels), each site $i$ is considered as a vector $\boldsymbol{a}_{i}=\left(a_{x i}, a_{y i}, a_{z i}\right)^{\mathrm{T}}$ relative to the center of the cluster, which is calculated from the arithmetic mean of the coordinates of all cluster voxels in each direction. The anisotropy of the permeability can be described by the orientation matrix $\boldsymbol{T}=\sum_{i=1}^{n} \boldsymbol{a}_{i} \boldsymbol{a}_{i}^{\mathrm{T}}$, where $\boldsymbol{a}_{i} \boldsymbol{a}_{i}^{\mathrm{T}}$ is the dyadic product of the site vector $\boldsymbol{a}_{i}$ and the transposed site vector $\boldsymbol{a}_{i}^{\mathrm{T}}$. The orientation matrix has 3 eigenvalues $\tau_{1}<$ $\tau_{2}<\tau_{3}$ and corresponding eigenvectors $\boldsymbol{u}_{1}, \boldsymbol{u}_{2}, \boldsymbol{u}_{3}$. Isotropy index $I=\tau_{1} / \tau_{3}$ and elongation index $E=1$ $-\tau_{2} / \tau_{3}$ are defined to estimate the intensity of anisotropy. $I \rightarrow 1$ and $E \rightarrow 0$ imply isotropic permeability. To allow comparison among different volumes and shapes, the components of a site vector are normalized by its volume size in the corresponding directions. The orientation matrix and its eigenvalues are normalized by the sum of the eigenvalues. 


\subsection{Stochastic Analysis}

[11] To understand the effect of scale and to extrapolate microscale to macroscale, a stochastic analysis is necessary. The so-called local porosity theory [Hilfer, 1992, 2002; Biswal et al., 1998] is used as a stochastic model. The sample to be analyzed by the local porosity theory should be a rectangular parallelepiped of a simple cubic lattice, whose side lengths are $M_{1}, M_{2}$ and $M_{3}$ in units of the lattice constant $a$, i.e., the resolution of the images. Let $\mathrm{K}(\boldsymbol{x}, L)$ denote a subvolume which is a cube of side length $L$ and whose center is located at the lattice vector $\boldsymbol{x}$. The subvolume acts as a moving window [Williams and Baxter, 2006] and scans through the whole model. The moving step can be equal to the lattice constant or an integer multiple of the lattice constant. Statistical results can be derived from a series of calculations of porosity, percolation and anisotropic permeability of each subvolume for different subvolume sizes $L$.

[12] The local porosity distribution $\mu(\phi, L)$ is defined as

$$
\mu(\phi, L)=\frac{1}{m} \sum_{x} \delta(\phi-\phi(x, L)),
$$

where $\delta$ denotes the Dirac $\delta$ function, $\phi(x, L)$ is the local porosity in the subvolume $\mathrm{K}(\boldsymbol{x}, L), m$ is the total number of subvolumes with side length $L$.

[13] The local percolation probability in the $\alpha$ direction is defined through

$$
\lambda_{\alpha}(\phi, L)=\frac{\sum_{x} \Lambda_{\alpha}(x, L) \delta_{\phi, \phi(x, L)}}{\sum_{x} \delta_{\phi, \phi(x, L)}}
$$

where

$$
\begin{aligned}
\delta_{\phi, \phi(x, L)} & =\left\{\begin{array}{ll}
1 & \text { if } \phi=\phi(x, L) \\
0 & \text { otherwise }
\end{array},\right. \text { and } \\
\Lambda_{\alpha} & = \begin{cases}1 & \text { if percolating in } \alpha \text {-direction } \\
0 & \text { otherwise }\end{cases}
\end{aligned}
$$

$\alpha=x, y$, or $z$ denote the direction along every axis, and $\alpha=3$ represents the percolation along all three directions.

[14] For the analysis of the anisotropy of permeability, similar to equation (1) we define the local anisotropy distributions as

$$
\chi(I, L)=\frac{1}{m^{\prime}} \sum_{x} \delta(I-I(x, L)),
$$

where $m^{\prime}$ is the total number of percolating subvolumes with side length $L, I$ represent 2 indices of anisotropic permeability, the isotropy index $I_{1}=I=\frac{\tau_{1}(\boldsymbol{x}, L)}{\tau_{3}(\boldsymbol{x}, L)}$ and the elongation index $I_{2}=E=$ $1-\frac{\tau_{2}(\boldsymbol{x}, L)}{\tau_{3}(\boldsymbol{x}, L)}$. Here $\tau_{i}(\boldsymbol{x}, L)$ are 3 normalized eigenvalues of the orientation matrix of a percolating cluster in subvolume $\mathrm{K}(\boldsymbol{x}, L)$ that satisfy $\sum_{i=1}^{3} \tau_{i}(\boldsymbol{x}, L)=1$. When more than one cluster is percolating in a subvolume, only the largest percolating cluster is considered for the statistical analysis.

\subsection{Parallelization of Stochastic Analysis}

[15] Generally, the analysis of porosity, percolation and anisotropy for a single volume can be calculated on a single processor in a short amount of time, e.g., $0.1-0.2 \mathrm{~s}$ for a volume of 1 million voxels. The total number of subvolumes $m$ in a stochastic analysis is given by

$$
m=\prod_{i=1}^{3}\left(\operatorname{INT}\left(\frac{M_{i}-L}{n}\right)+1\right),
$$

where $n$ represents the moving step in units of the lattice constant. When $n=L$, subvolumes are placed in nonoverlapping way, it might lead to fluctuation of results; for $n<L$, it is an overlapping placement and gives higher weight to the central region of the model. For a large data set and a small $n$, the computing time for a single processor becomes prohibitively large. To alleviate this problem, it is necessary to parallelize the calculation. The probabilities defined in equations (1)-(3) are dependent only on the summation of data within a moving window. By using the OpenMP programming techniques it was possible to quickly and easily augment the DO loops that move this window to move a team of windows, one for each available CPU. This parallelization splits up the most time-consuming part of the calculation.

\section{Data for Benchmarking}

[16] We use a synthetic quartz sandstone sample to verify the code and to demonstrate the method of visualization. The diameter of the sample is $5 \mathrm{~mm}$ which contains 543 slices with 2000 pixels in both $\mathrm{x}$ and $\mathrm{y}$ directions. The size of the data set is $2.01 \mathrm{~GB}$ and the resolution is $\sim 2.5$ micron. Figure 1 displays a slice of the original gray scale image and its corresponding binary image. A model to be analyzed should be a parallelepiped of $\mathrm{M}_{1} \times \mathrm{M}_{2} \times \mathrm{M}_{3}$ according to section 2.3. The larger the volume the better is the statistical information obtained. We have not included the boundary area of the sample because of noise in 

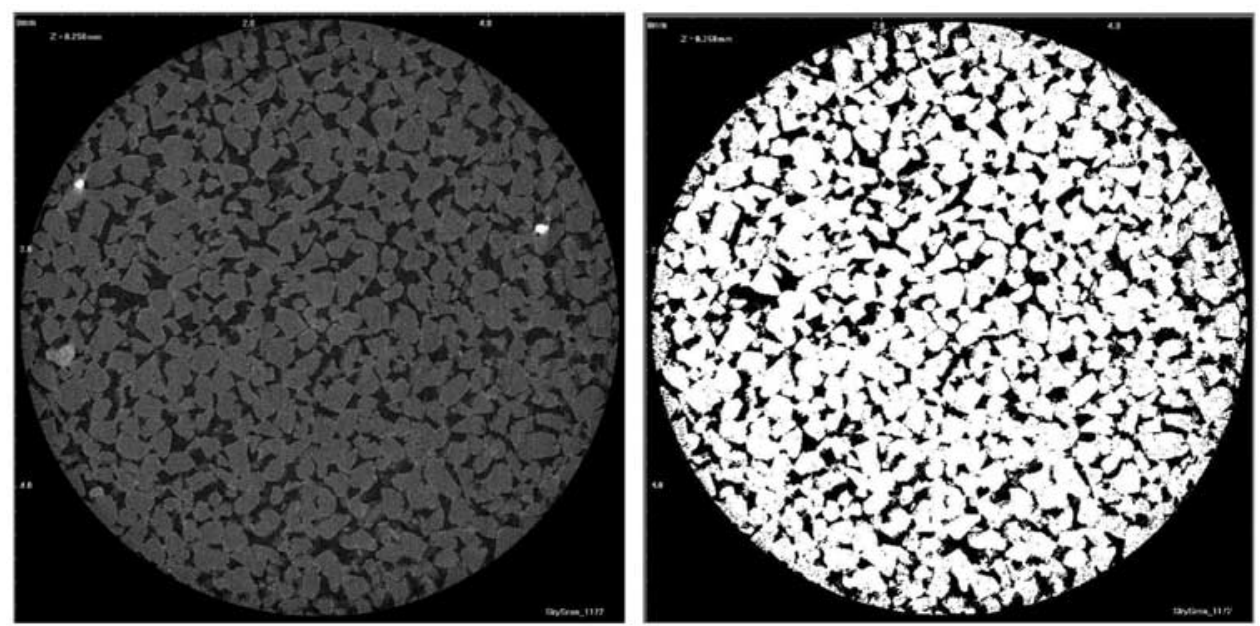

Figure 1. Gray scale image and binary image of a slice of the synthetic sandstone sample.

the perimeter. We were therefore limited with the largest possible realization to $0.75 \mathrm{~mm}$ away from the perimeter. This translates to the largest subset of $2 \times 2 \times 1 \mathrm{~mm}^{3}$ volume cropped from this sandstone sample. The gray scale histogram of the cropped volume and the 3-D microstructural model of solid after the preprocessing procedures are shown in Figure 2. Animation S1 was captured to show the 3-D pore structures. ${ }^{1}$

[17] In order to investigate the effects of resolution, we resample the raw image data by merging 2 and 4 voxels in every direction to get coarser data sets of resolution with 5 and $10 \mu \mathrm{m}$, respectively. These are referred to as medium- and low-resolution data, respectively. The original data is called high-resolution data.

\section{Results}

\subsection{Stochastic Analysis Results}

[18] For each resolution data set, a series of calculations are carried out for different subvolume sizes.

[19] Figure 3 shows the local porosity distributions for different subvolume sizes. Most curves show normal distribution, which conforms to the Central Limit Theorem that underpins the moving window method. As the subvolume size is increased the distribution of porosity becomes narrower and the average value of porosity, 0.2444 , is obtained. When the subvolume size is equal to or greater

${ }^{1}$ Auxiliary materials are available in the HTML. doi:10.1029/ 2008GC002358. than $800 \mu \mathrm{m}$, most subvolumes have porosity of 0.24 or 0.25 . Thus a subvolume with side length $800 \mu \mathrm{m}$ is sufficiently large to accurately estimate porosity. The different resolution data sets exhibit the same characteristics and yield the same result.

[20] Figure 4 presents the results of the local percolation probabilities. Figure 4a shows (1) the probabilities increase with increasing porosity; (2) the probabilities of percolation in $\mathrm{x}, \mathrm{y}$ and $\mathrm{z}$ directions are similar, which means the sample is almost isotropic; and (3) percolation in all 3 directions has lower probability than those one directional probabilities. Figure $4 \mathrm{~b}$ shows (1) the local percolation probabilities also increase with increasing subvolume size and (2) when the subvolume size is greater than $300 \mu \mathrm{m}$, the probabilities of percolation in 3 directions are almost $100 \%$. We also notice in Figure $4 \mathrm{~b}$ that some curves have a small range of porosity. It is because the denominator of right-hand side of equation (2) is zero, which is correspondent to $\mu(\phi, L)=0$, thus $\lambda_{\alpha}(\phi, L)$ has no solution outside this range for those subvolume sizes. Figure $4 \mathrm{c}$ compares the probabilities of different resolution data sets for porosity of 0.24 . Although the resolution appears to slightly influence the local percolation probability, i.e., higher-resolution data sets have higher percolation probabilities for small subvolume sizes, there is no difference when the subvolume size is greater than $300 \mu \mathrm{m}$, where all subvolumes are definitely percolating in 3 directions for all high-, medium- and low-resolution data. These results imply that for a statistical assessment of percolation the minimum representative elementary volume is $300 \mu \mathrm{m}$ of side length. 


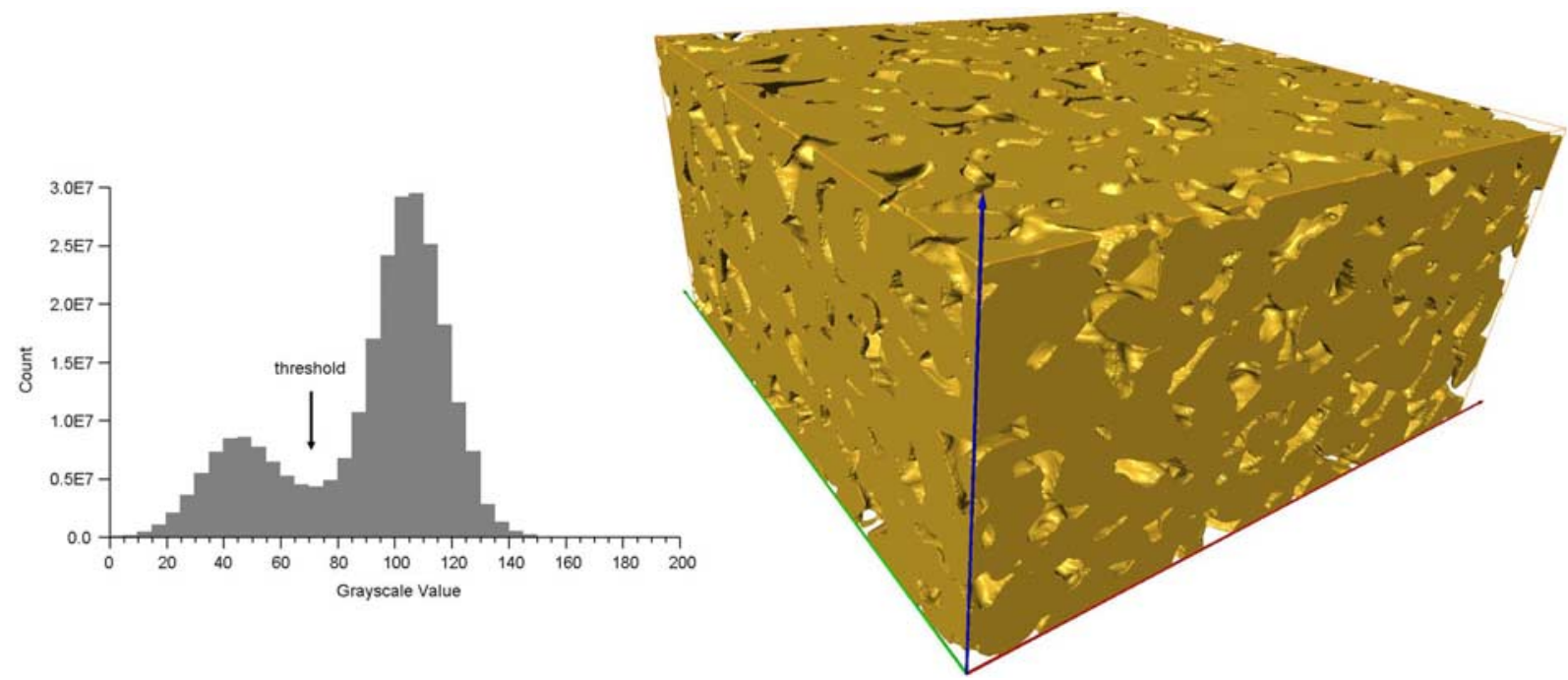

Figure 2. Gray scale histogram and 3-D solid structure of the volume in size of $2 \times 2 \times 1 \mathrm{~mm}^{3}$.

[21] Figure 5 presents the results of local anisotropy distributions. Figure 5a shows that the maximum probability locates in the low isotropy index for small subvolume sizes, i.e., locates in $I=0.14$ for $L=100 \mu \mathrm{m}$, indicating a strong anisotropy. As the subvolume size increases, the probability increases and gives a higher isotropy index. The locus of the maximum probability asymptotically equals to an isotropy index of 0.94 . In Figure 5b, the elongation index asymptotically approaches 0.03 when the subvolume size increases. These two indices indicate that the sandstone sample is near isotropic when the subvolume size is large enough, i.e., equal to or larger than $800 \mu \mathrm{m}$. For smaller subvolumes the permeabilities have strong anisotropy. Again, this conclusion is the same for the different resolution data sets.

[22] As a validation of the porosity and the anisotropy estimates, we compare our results with the experimental data and scanning electron microscope (SEM) images.

[23] The Helium porosimetry reveals that the bulk porosity of the sample is approximately $28.5 \%$, which is about 4 porosity unit higher than the porosity calculated from the micro-CT images. This discrepancy between these two porosity results was assessed by using Scanning Electron Microscope (SEM) point counting analysis and additional nanometer resolution field emission SEM (Figure 6). As shown in Figure 6, there are micropores in the sandstone sample with dimensions less than 2.5 microns, which is the resolution of the X-ray CT images. These micropores exist in three different sites: (1) cavities within the calcite cement, (2) microgaps between the calcite rims and the quartz grains, and (3) microfractures in the quartz sand grains. Because of the resolution limits of the X-ray CT scans these micropores are labeled as solids and are therefore not detected. This leads to a systematic underestimate of the porosity.

[24] For additional benchmarking of our results we used high-resolution point counting with the AutoGeoSEM approach developed at CSIRO Exploration and Mining. This analysis was done on a 2-D slice through the sample with 1.6 microns local resolution. This is $64 \%$ higher in resolution than that of the X-ray CT image. In practice the X-ray pore identification is lower than the theoretical limit of 2.5 microns because it must span several voxels in at least one dimension for reliable detection [Ketcham, 2005]. With the SEM technique a total of 13860 points were examined. The porosity from the SEM point counting is $27.3 \%$. This is closer to the Helium measurement by $2.8 \%$. This is

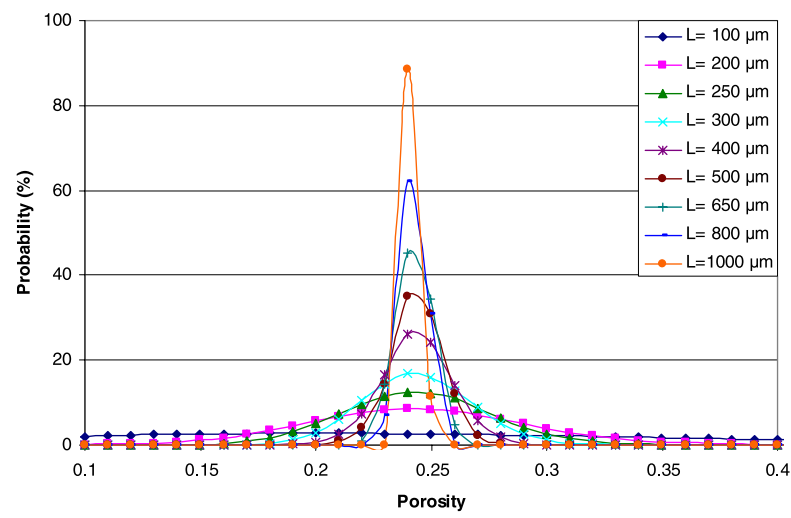

Figure 3. Local porosity distribution of the model. 

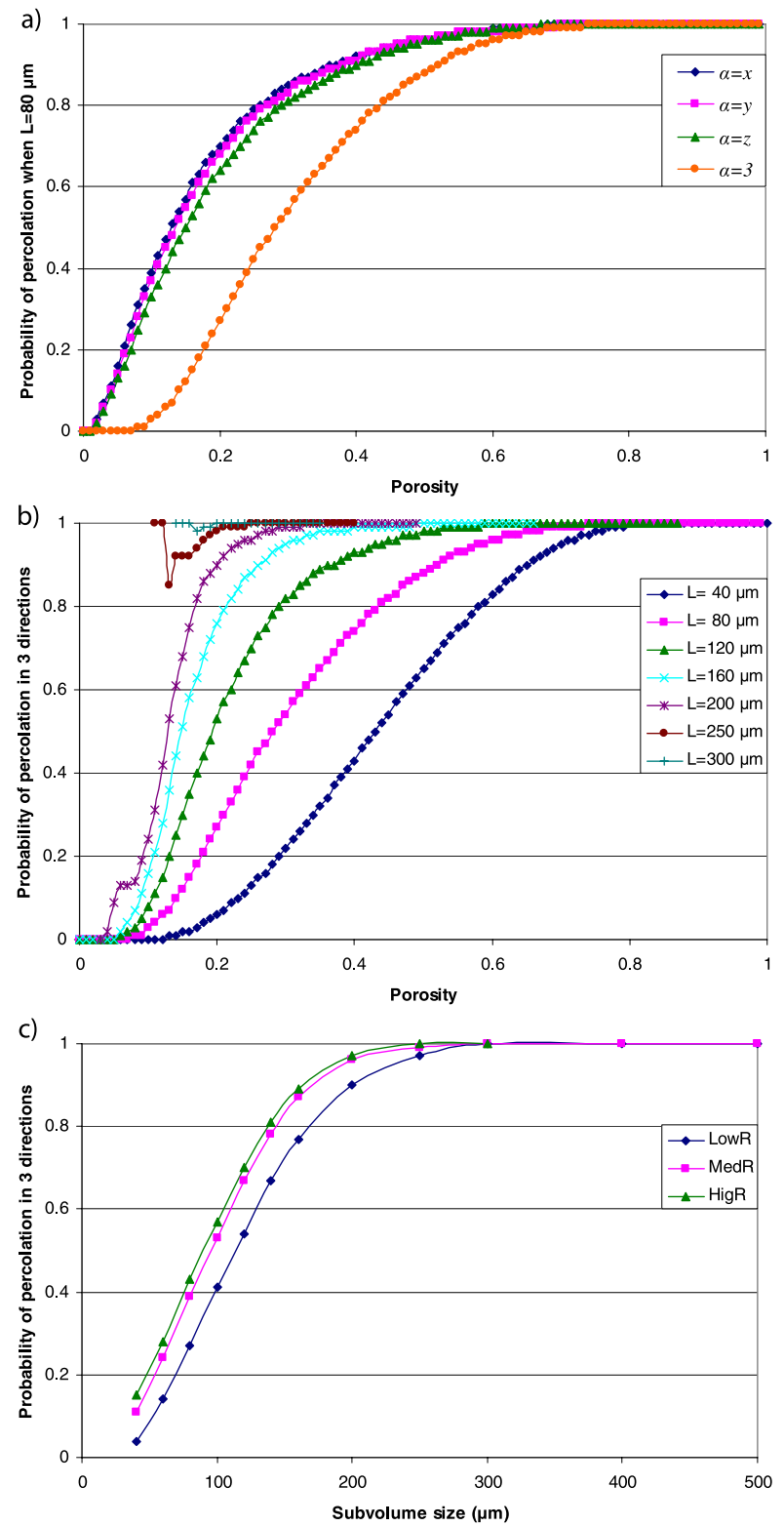

Figure 4. Results of local percolation probabilities. (a) Probability ( $\alpha=x, y, z, 3)$ versus porosity, $L=80 \mu \mathrm{m}$ of medium-resolution data. (b) Probability $(\alpha=3)$ versus porosity for different $L$, medium-resolution data. (c) Probability $(\alpha=3)$ versus subvolume size for different resolutions, $\phi=0.24$.

a clear indication that a higher-resolution image can detect more pores. We performed an additional field emission SEM analysis of the cement with nanometer resolution. This technique does not allow a quantitative analysis of pore space. However, visual examination of this nanometer resolution SEM image indicates the presence of submicron pores in the cement not resolved by the SEM point counting. We conclude that the discrepancy between experimental and image anal- ysis can therefore be entirely attributed to resolution limits.

[25] Concerning the anisotropy assessment the laboratory measurements suggest that the synthetic sandstone sample has an almost isotropic Klinkenberg permeability of 4.5 Darcy. The conclusion of near isotropic permeability is consistent with the results of the percolation analysis based on the CT images.

[26] The above comparison between experimental and computational results suggests that the code can adequately describe porosities and permeability anisotropy within the resolution limits of the image data supplied.

\subsection{Visualization}

[27] In addition to the probabilities of porosity, percolation and anisotropy discussed in the previous section, we would like to assess the microstructure with a visual approach. Figure 2 and Animation S1 illustrate the microstructure of the model. Next we will illustrate the percolation and anisotropy of permeability.
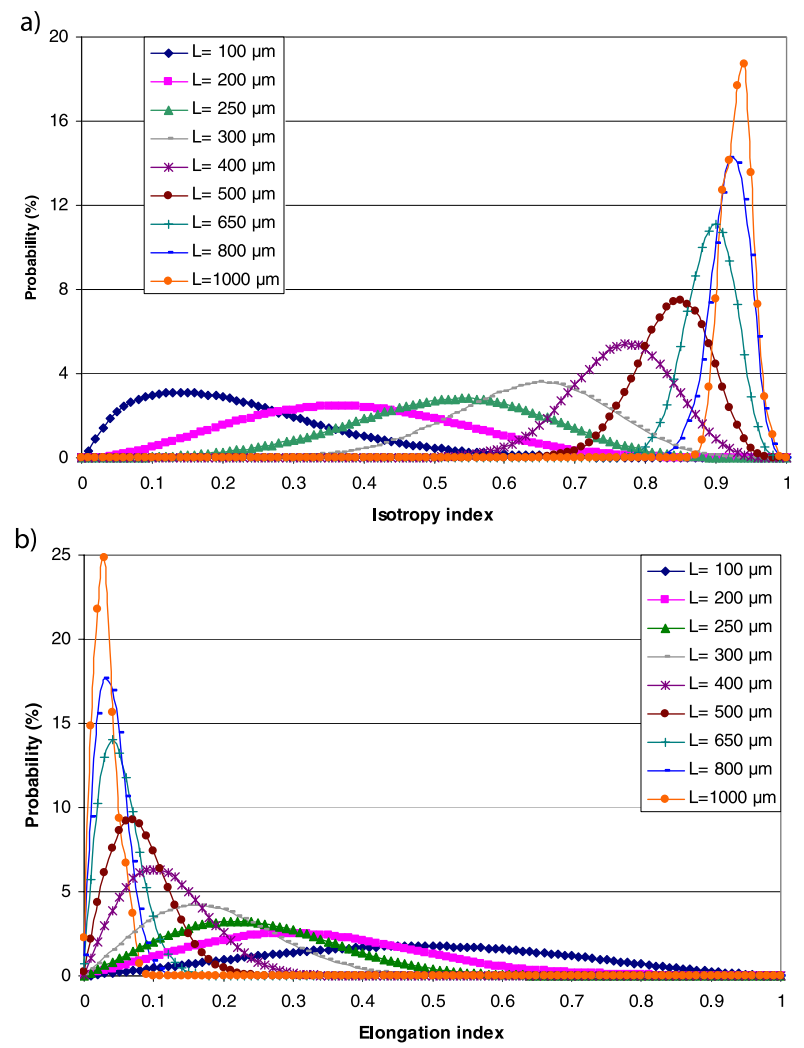

Figure 5. Probabilities of (a) isotropy index and (b) elongation index. 

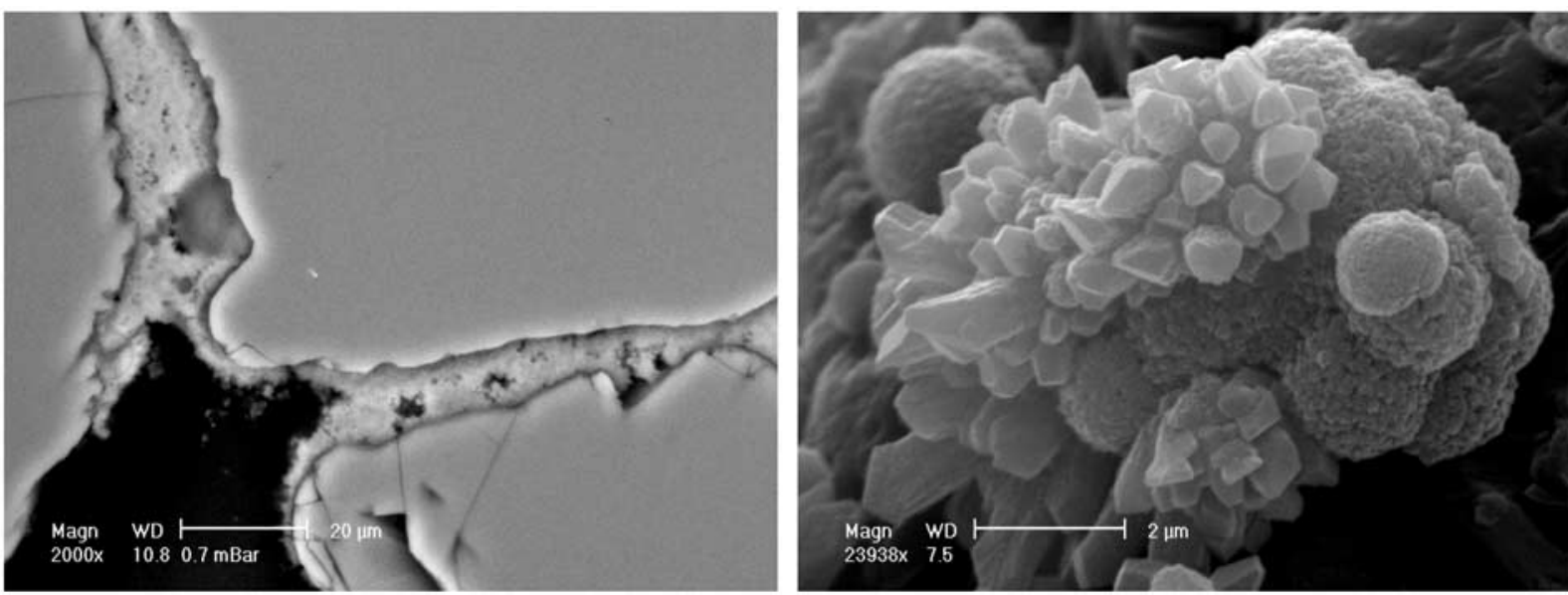

Figure 6. SEM images of calcite cement rims around quartz grains showing the presence of micropores and the porous nature of the calcite crystals that are beyond the resolution of X-ray CT used in this investigation.

[28] Labeling clusters is the key procedure for the percolation analysis. Visualization of clusters of a volume not only illustrates the structure and connectivity of pores, but also checks the result of percolation and verifies the codes. We use a finite element preprocessor $\left(\mathrm{GID}^{\mathbb{R}}\right)$ to visualize the clusters. Voxels are recognized as hexahedral elements and labels of different clusters are treated as numbers of material of elements. Clusters can be displayed selectively in this approach. Figure 7 illustrates clusters of pores with different volume sizes. Clusters are displayed in different colors. For ease of visual interpretation the color scale is rendered by 20 different colors distinguishing the largest clusters. Small clusters are rendered in a single color. It is considered that those isolated clusters do not contribute to fluid flow, on the basis of the identification of current image resolution.

[29] The anisotropic permeability of a subvolume is represented by the tensor of anisotropy and illustrated by an ellipsoid corresponding to the tensor. In Figure 8 tensors are shown in nonoverlapping subvolumes with subvolume sizes of $100 \mu \mathrm{m}$ and $500 \mu \mathrm{m}$, respectively. For the small subvolume size (Figure 8a), tensors of anisotropy have varying shapes and their orientations are random distributions. In contrast, for the large subvolume size (Figure 8b), the shape of tensors is more spherical, indicating a tendency toward isotropy. Furthermore, the distribution of orientations can be captured by a stereographic projection of the principal axes of the tensors. Figure 9 gives the stereographic projection of the maximum principal axes of the tensors corresponding to Figure 8.

[30] The tensor of anisotropic permeability is related to the structure of the percolating cluster in the subvolume as illustrated in Figure 10. We zoom in to the lower corner of Figure 8a and focus on the range of $x=1-400, y=1-400$ and $z=1-100 \mu \mathrm{m}$. With a subvolume size of $100 \mu \mathrm{m}$, there should be 16 nonoverlapping subvolumes. Only 14 ellipsoids are shown because there are 2 subvolumes that are not percolating. Five arbitrarily chosen ellipsoids of tensor are labeled as $\mathrm{A}-\mathrm{E}$, the corresponding pore clusters are shown in the inset boxes also labeled A-E. Both representations are equivalent. However, the ellipsoidal representation allows a quantification of the anisotropy. We would like to point out that on the basis of the percolation theory, the anisotropic permeability is different from that calculated from all pores. A particular example is clearly illustrated in the inset box D. While pores are distributed almost equally in $\mathrm{x}$ and $\mathrm{y}$ directions, only one pore cluster is percolating in $\mathrm{x}$ direction, thus the anisotropy of permeability is also oriented in $\mathrm{x}$ direction.

\subsection{Computing Speed-Up Test}

[31] The performance of parallelization is measured by the speed-up, which is defined as the ratio of wall time of 1-CPU to the wall time of $\mathrm{N}$ CPUs. We tested the speed-up of our codes in different calculations. Figure 11 shows the speedup curve of CPUs from 2 to 32 for the model size of $400 \times 400 \times 200$ (medium-resolution data) with a subvolume size $L=40 \mu \mathrm{m}$. In Figure 11, the shaded area is the region between the upper bound (the ideal speed-up) and the lower bound (allowed on our supercomputer for minimum speed-up) defined by the square root of the number of CPUs. it can be seen from Figure 11, when 2 or 4 CPUs are used the speed-up is linear, while with 8 and 16 CPUs the speed-up relatively drops, but 


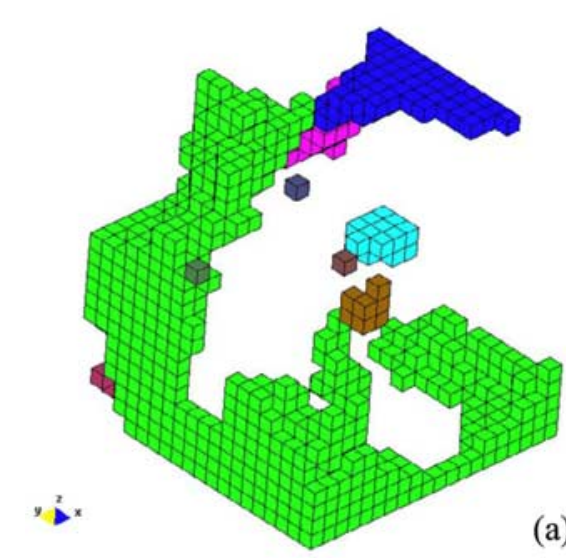

(a)

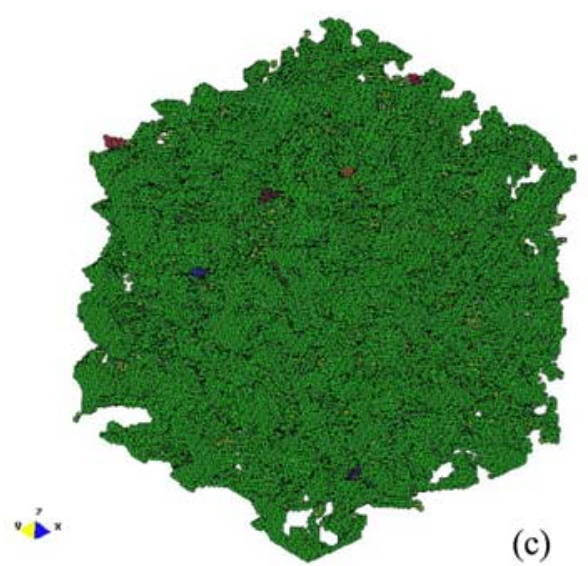

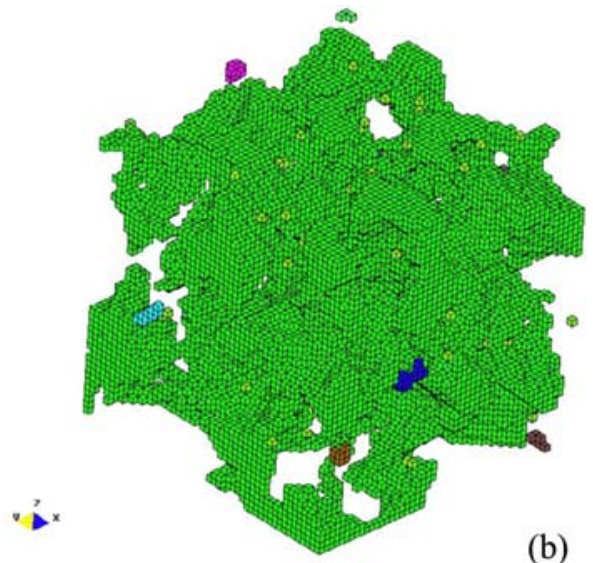

(b)

(d)

Figure 7. Pore clusters in different subvolume sizes. (a) Pore clusters in subvolume from $(1,1,1)$ to $(20,20,20)$. (b) Pore clusters in subvolume from $(1,1,1)$ to $(50,50,50)$. (c) Pore clusters in subvolume from $(1,1,1)$ to $(100,100,100)$. (d) Small pore clusters of Figure $7 \mathrm{c}$; the largest cluster is hidden.

improves again with 24 or 32 CPUs. This varying behavior is typical for the cache coherent NonUniform Memory Access architecture cache effect. This effect does not only depend on the number of processors but also on the size of the models and subvolumes. Thus, for an unknown problem it is impossible to estimate the performance a priori. It is generally necessary to do a performance test on a case by case study. However, our tests have demonstrated that all the speed-ups are acceptable, i.e., tested speed-up is above the minimum line.

\section{Discussion}

[32] In this paper, we offered a comprehensive analysis of the porosity, percolation and anisotropic permeability by using X-ray micro-CT scan images and mathematical methods. The results from the synthetic sandstone sample demonstrated the viability of the extended local porosity theory. With the additional visualization, the characteristics of microstructures can be captured quantitatively and visually. The method developed here is an appropriate method to extract the stochastic geometry properties of any microstructures.

[33] The new method gives the definition of anisotropy characteristics in a natural extension to Hilfer's classical local porosity theory. Hilfer's porosity distribution and percolation probability are extended by anisotropy distribution functions. These describe the intensity of percolation of different directions and provide much more detailed information for fluid flow in a 3-D space. On the basis of the percolation theory and cluster labeling, the anisotropy of permeability is only related to the percolating pore cluster, all nonpercolating or isolated pore clusters which do not contribute to fluid flow are not involved in the calculation of anisotropy.

[34] Our analysis not only allows the identification of a representative volume element which can be 

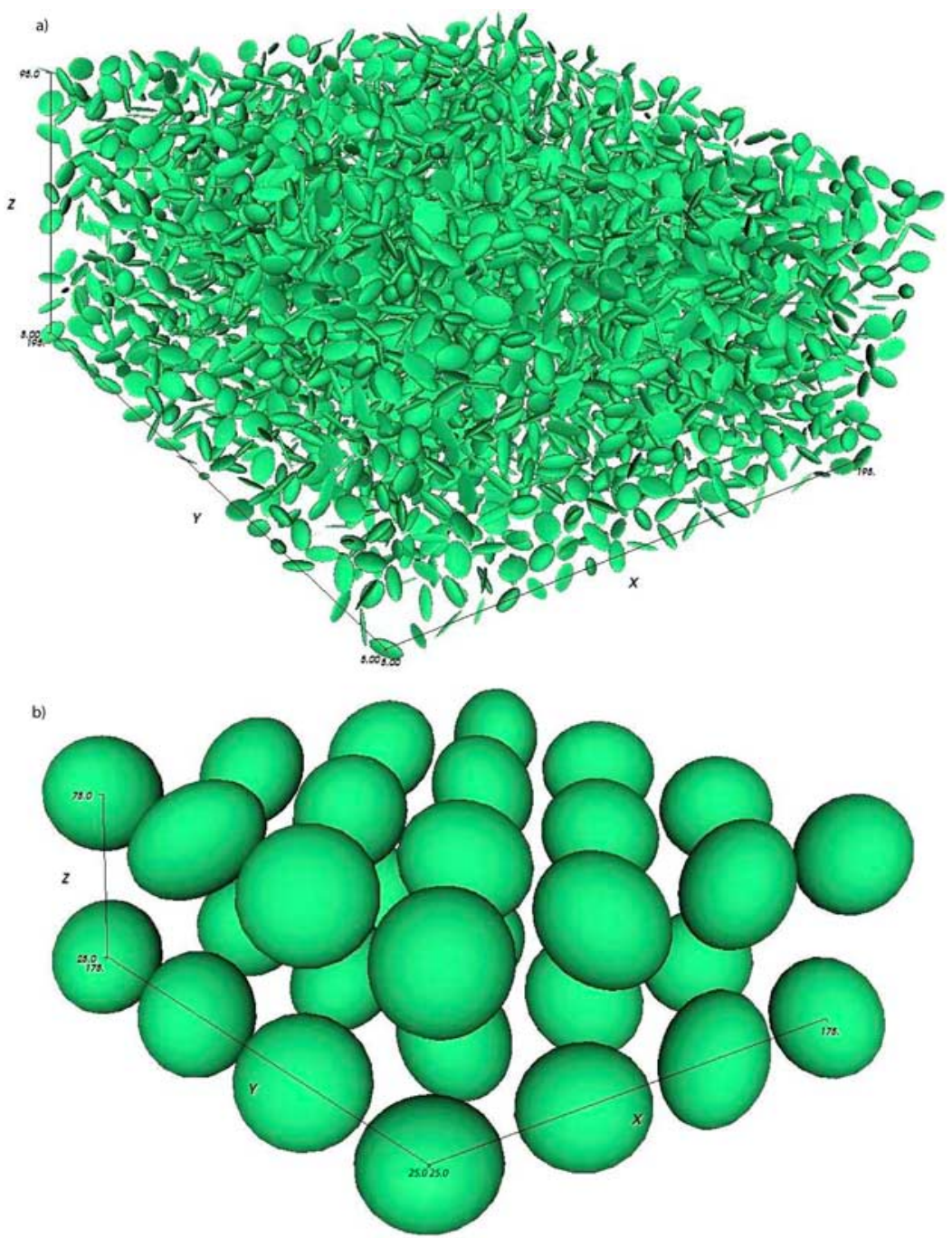

Figure 8. Tensors of anisotropic permeability in nonoverlapping subvolumes (unit of axes is voxel). (a) Subvolume size is $100 \mu \mathrm{m}$. (b) Subvolume size is $500 \mu \mathrm{m}$.
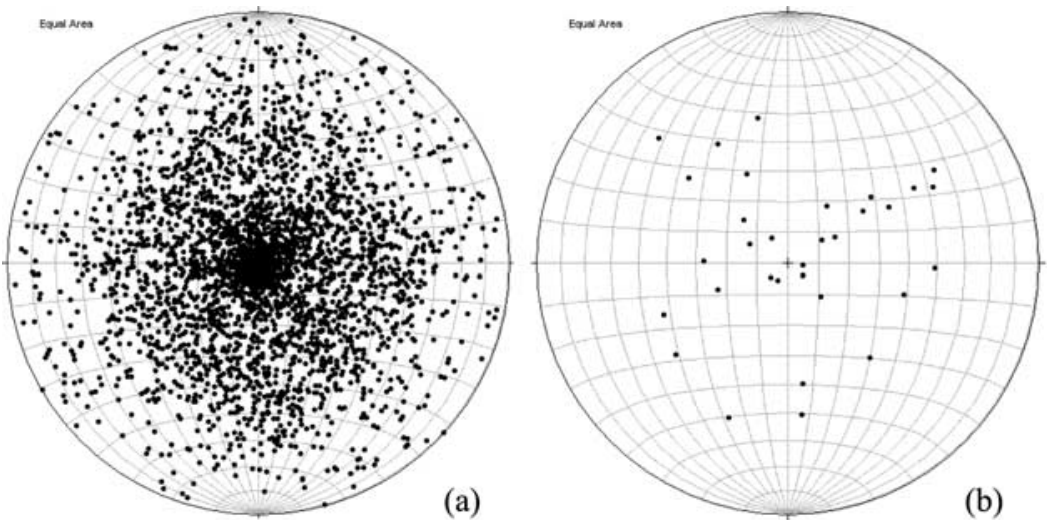

Figure 9. The maximum principal axes of tensors in the stereographic net. (a) Subvolume size is $100 \mu \mathrm{m}$. (b) Subvolume size is $500 \mu \mathrm{m}$. 


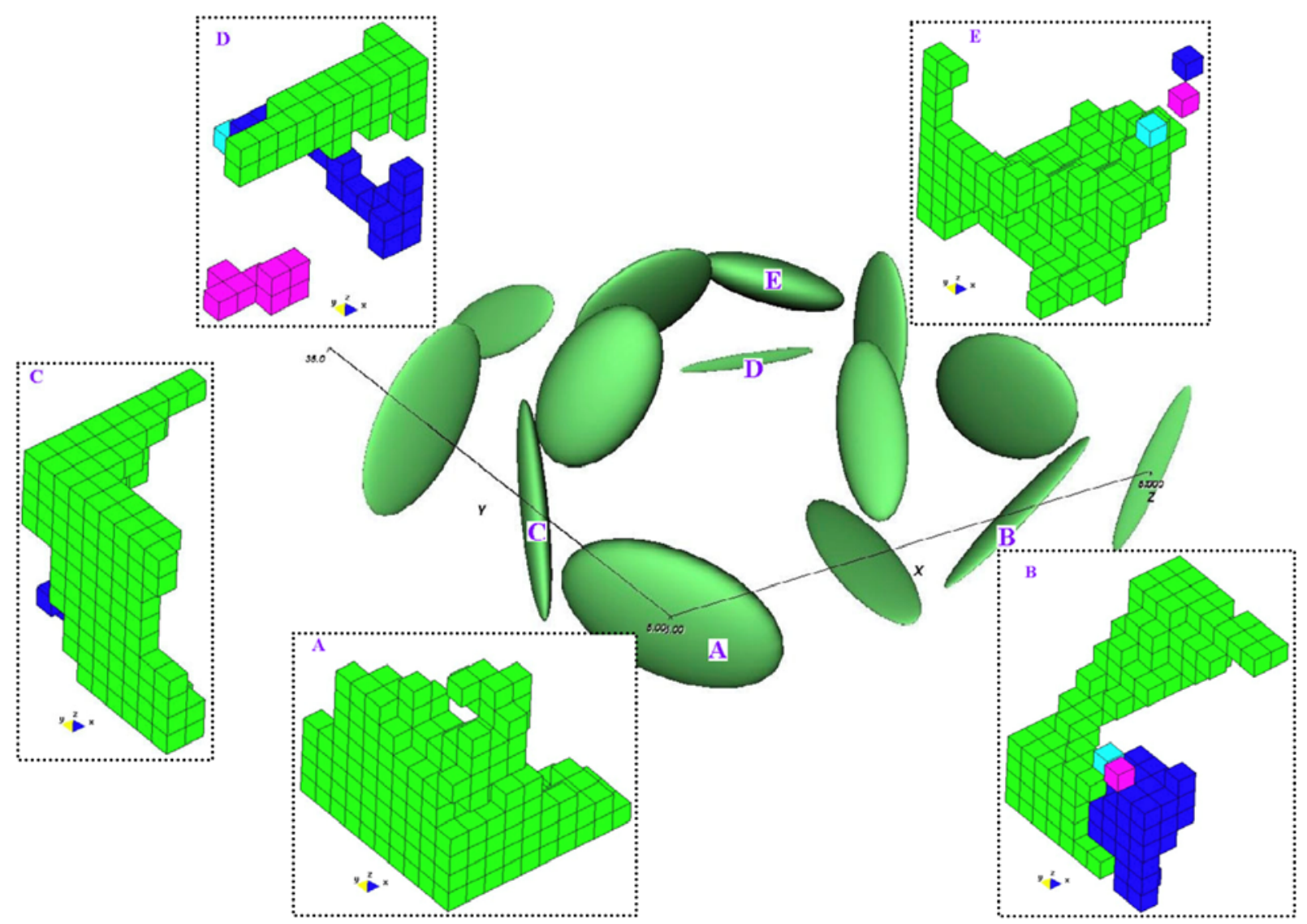

Figure 10. Comparison of tensors of permeability and pore clusters.

used for feeding into a homogenization approach such as in finite elements, but also allows the calculation of important scaling laws such as critical exponents and site percolation threshold. Furthermore, the method can also be used as a building block for a heterogeneous multiscale method [E and Engquist, 2003]. The heterogeneous multiscale method is one of many methods to derive a generalized numerical solution technique for multiscale processes in heterogeneous media. It relies on a macroscale solver and supplies missing numerical data from microscale models. Microstructure analysis based on the present method can feed observation data to the microscale model. The approach is suitable for quantification of microscale to macroscale transitions. This analysis thus provides the first step in a multiscale assessment and quantification of physical properties of materials.

[35] The parallelization of the code makes it possible to handle data sets of gigabyte sizes, an increment of $2 \mathrm{~GB}$ can be added per additional processor. The parallel performance is limited by the OpenMP architecture. Generally, the efficiency of speed-up becomes lower when more processors are used. We suggest using less than 32 processors. Future enhancements can be gained from MPI parallelization, although OpenMP is ideal for this problem if less than 32 processors are available.

[36] From the example of the synthetic sandstone, the representative volume element of around $1 \mathrm{~mm}^{3}$ is enough. In addition, the resolution sensitivity analysis allows an identification of the lowest permissible resolution. This low-resolution data

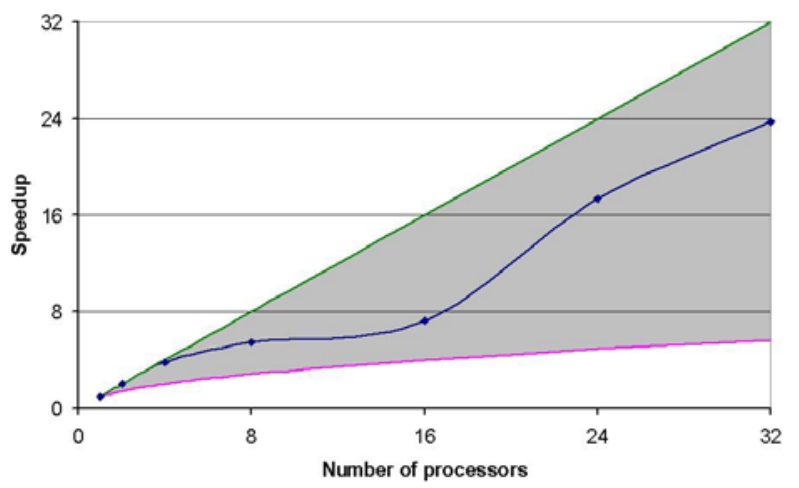

Figure 11. Computing speed-up chart. 
can be used for further forward numerical modeling of this sample because the values of porosity, percolation and anisotropic permeability in 3 resolutions do not differ significantly from each other. Thus the method suggested here allows an assessment of the lowest resolution for numerical forward modeling of microscale processes.

[37] A preliminary application of the method was conducted in a natural mylonite sample, which displays an extreme anisotropy of the microstructure of pores [Liu et al., 2009]. Results of subsamples in the scale of $0.6-1 \mathrm{~mm}$ of the mylonite showed that (1) in the margin of the localized shear zone, random and irregularly distributed pores are not percolating in the subsample scale and (2) in the center of the shear zone, there are numerous nonpercolating smaller pores and two percolating directions, with strongly anisotropic permeability. Further study revealed that a dynamic permeability related to cavitation along grain boundaries is maintained [Fusseis et al., 2009].

[38] Note, that the percolation analysis does not equate to a permeability estimate but it is the first step toward such an estimate from CT images. The analysis will be continued through forward simulation techniques in order to obtain permeability estimates as function of pressure, deviatoric stress, temperature and chemical composition of solids and fluids. We have shown an example for analysis of a porosity network, however, the statistical method is suitably general that it can be used for any microstructural analysis such as damage, grain size, dislocation, crack, chemical species distributions etc. The stochastic geometry approach will allow an inclusion of microscale material properties and processes into the computations of largescale processes. Such calculations will be useful in many disciplines, e.g., from earthquake simulations and geodynamics applications at the largest scale down to petroleum engineering, geothermal and mining applications at intermediate scale and laboratory analysis at the smallest scale.

\section{Acknowledgments}

[39] This project was funded through the Premier's Fellowship Program of the Western Australian Government and the CSIRO OCE Postdoctoral Program. The X-ray micro-CT data set was provided by the Microbially Enhanced Oil Recovery project of CSIRO's Wealth from Ocean Flagship. We are grateful to iVEC for technical support and access to its visualization facilities and Altix supercomputer, Michael Verrall of CSIRO Exploration and Mining for helping with the point counting using AutoGeoSEM, and Bibudhananda
Biswal for his help in local porosity theory. This paper benefits from discussion with Florian Fusseis of the University of Western Australia and Thomas Poulet, Heather Sheldon, and Yanhua Zhang of CSIRO Exploration and Mining.

\section{References}

Arns, C. H., M. A. Knackstedt, W. V. Pinczewski, and W. B. Lindquist (2001), Accurate estimation of transport properties from microtomographic images, Geophys. Res. Lett., 28, 3361-3364, doi:10.1029/2001GL012987.

Biswal, B., and R. Hilfer (1999), Microstructure analysis of reconstructed porous media, Physica A, 266, 307-311, doi:10.1016/S0378-4371(98)00607-4.

Biswal, B., C. Manwart, and R. Hilfer (1998), Three-dimensional local porosity analysis of porous media, Physica A, 255, 221-241, doi:10.1016/S0378-4371(98)00111-3.

E, W., and B. Engquist (2003), The heterogeneous multiscale methods, Commun. Math. Sci., 1, 87-133.

Fusseis, F., K. Regenauer-Lieb, J. Liu, R. M. Hough, and F. De Carlo (2009), Creep cavitation and dissolution establish a granular fluid pump in ductile shear zones, Nature, doi:10.1038/nature08051, in press.

Hilfer, R. (1992), Local porosity theory for flow in porous media, Phys. Rev. B, 45, 7115-7121, doi:10.1103/PhysRevB.45.7115.

Hilfer, R. (2002), Review on scale dependent characterization of the microstructure of porous media, Transp. Porous Media, 46, 373-390, doi:10.1023/A:1015014302642.

Hoshen, J., and R. Kopelman (1976), Percolation and cluster distribution. 1. Cluster multiple labeling technique and critical concentration algorithm, Phys. Rev. B, 14, 3438-3445, doi:10.1103/PhysRevB.14.3438.

Jerram, D. A., and M. D. Higgins (2007), 3D analysis of rock textures: Quantifying igneous microstructures, Elements, 3, 239-245, doi:10.2113/gselements.3.4.239.

Ketcham, R. A. (2005), Three-dimensional grain fabric measurements using high-resolution X-ray computed tomography, J. Struct. Geol., 27, 1217-1228, doi:10.1016/ j.jsg.2005.02.006.

Ketcham, R. A., and G. J. Iturrino (2005), Nondestructive high-resolution visualization and measurement of anisotropic effective porosity in complex lithologies using high-resolution X-ray computed tomography, J. Hydrol., 302, 92-106, doi:10.1016/j.jhydrol.2004.06.037.

Knackstedt, M. A., C. H. Arns, M. Saadatfar, T. J. Senden, A. Limaye, A. Sakellariou, A. P. Sheppard, R. M. Sok, W. Schrof, and H. Steininger (2006), Elastic and transport properties of cellular solids derived from 3D tomographic images, Proc. R. Soc. A, 462, 2833-2862.

Liu, J., K. Regenauer-Lieb, and F. Fusseis (2009), Stochastic analysis of percolation and anisotropic permeability from micro-tomography and an application to mylonite, $A d v$. Geosci., in press.

Manwart, C., U. Aaltosalmi, A. Koponen, R. Hilfer, and J. Timonen (2002), Lattice-Boltzmann and finite-difference simulations for the permeability for three-dimensional porous media, Phys. Rev. E, 66, 016702, doi:10.1103/ PhysRevE.66.016702.

Regenauer-Lieb, K., et al. (2008), First steps towards a multiscale earth system, in Advances in Geocomputing, edited by H. Xing, pp. 1-26, Springer, Berlin.

Stauffer, D., and A. Aharony (1994), Introduction to Percolation Theory, 2nd ed., Taylor and Francis, London. 
Widjajakusuma, J., B. Biswal, and R. Hilfer (1999), Quantitative prediction of effective material properties of heterogeneous media, Comput. Mater. Sci., 16, 70-75, doi:10.1016/ S0927-0256(99)00047-6.
Williams, T. O., and S. C. Baxter (2006), A framework for stochastic mechanics, Probab. Eng. Mech., 21, 247-255, doi:10.1016/j.probengmech.2005.10.002. 